\title{
ODDS THEOREM WITH MULTIPLE SELECTION CHANCES
}

\author{
KATSUNORI ANO, ${ }^{*}$ Institute of Applied Mathematics \\ HIDEO KAKINUMA ** AND \\ NAOTO MIYOSHI, ${ }^{* * *}$ Tokyo Institute of Technology
}

\begin{abstract}
We study the multi-selection version of the so-called odds theorem by Bruss (2000). We observe a finite number of independent $0 / 1$ (failure/success) random variables sequentially and want to select the last success. We derive the optimal selection rule when $m(\geq 1)$ selection chances are given and find that the optimal rule has the form of a combination of multiple odds-sums. We provide a formula for computing the maximum probability of selecting the last success when we have $m$ selection chances and also provide closed-form formulae for $m=2$ and 3. For $m=2$, we further give the bounds for the maximum probability of selecting the last success and derive its limit as the number of observations goes to $\infty$. An interesting implication of our result is that the limit of the maximum probability of selecting the last success for $m=2$ is consistent with the corresponding limit for the classical secretary problem with two selection chances.
\end{abstract}

Keywords: Optimal stopping; selecting the last success; multiple selection chances

2010 Mathematics Subject Classification: Primary 60G40

Secondary 62L15

\section{Introduction}

For a positive integer $N$, let $X_{1}, X_{2}, \ldots, X_{N}$ denote independent $0 / 1$ random variables defined on a probability space $(\Omega, \mathcal{F}, \mathrm{P})$. We observe these $X_{i}$ s sequentially and claim that the $i$ th trial is a success if $X_{i}=1$. The problem lies in finding a rule $\tau \in \mathcal{T}$ to maximize the probability of selecting the last success, where $\mathcal{T}$ is the class of all selection rules such that $\{\tau=j\} \in \sigma\left(X_{1}, X_{2}, \ldots, X_{j}\right)$, that is, the decision of whether to select the $j$ th success depends on the information up to $j$. Let $\mathcal{N}=\{1,2, \ldots, N\}$, and let $p_{i}=\mathrm{P}\left(X_{i}=1\right)$ and $q_{i}=1-p_{i}=\mathrm{P}\left(X_{i}=0\right)$ for $i \in \mathcal{N}$. In addition, let $r_{i}, i \in \mathcal{N}$, denote the odds of the $i$ th trial; that is, $r_{i}=p_{i} / q_{i}$, where we set $r_{i}=+\infty$ if $p_{i}=1$. When exactly one selection chance was allowed, Bruss [3] solved the problem with elegant simplicity as follows.

Proposition 1.1. (Theorem 1 of [3].) Suppose that exactly one selection chance is given in the problem above. Then, the optimal selection rule $\tau_{*}^{(1)}$ selects the first success after the sum of

\footnotetext{
Received 11 May 2010; revision received 30 September 2010.

* Postal address: Department of Applied Probability, Institute of Applied Mathematics, Asakusabashi, Taito-ku, Tokyo 111-0053, Japan. Email address: kano@iapm.jp

** Postal address: Department of Mathematical and Computing Sciences, Tokyo Institute of Technology, 2-12-1-W8-52 Ookayama, Meguro-ku, Tokyo 152-8552, Japan.

*** Postal address: Department of Mathematical and Computing Sciences, Tokyo Institute of Technology, 2-12-1-W852 Ookayama, Meguro-ku, Tokyo 152-8552, Japan. Email address: miyoshi@is.titech.ac.jp
} 
the future odds becomes less than 1; that is

$$
\begin{aligned}
\tau_{*}^{(1)} & =\min \left\{i \geq i_{*}^{(1)}: X_{i}=1\right\}, \\
i_{*}^{(1)} & =\min \left\{i \in \mathcal{N}: \sum_{j=i+1}^{N} r_{j}<1\right\},
\end{aligned}
$$

where $\min (\varnothing)=+\infty$ and $\sum_{j=a}^{b} \cdot=0$ when $b<$ a conventionally. Furthermore, the maximum probability of 'win' (selecting the last success) is given by

$$
P^{(1)}(\text { win })=P_{N}^{(1)}\left(p_{1}, \ldots, p_{N}\right)=\prod_{k=i_{*}^{(1)}}^{N} q_{k} \sum_{k=i_{*}^{(1)}}^{N} r_{k} .
$$

This result, referred to as the sum-the-odds theorem, or the odds theorem for short, is attractive because it can be applied to many basic optimal stopping problems, such as betting, the classical secretary problem (CSP), and the group-interview secretary problem proposed by Hsiau and Yang [11]. Bruss [3] also proved that $P^{(1)}$ (win) in (1.3) is bounded below by $R^{(1)} \mathrm{e}^{-R^{(1)}}$ with $R^{(1)}=\sum_{j=i^{(1)}}^{N} r_{j}$. Remarkably, in [4], he found that it is bounded below by $\mathrm{e}^{-1}$ when $\sum_{j=1}^{N} r_{j} \geq 1$. These results generalize the known lower bounds for the CSP, where each $p_{i}$ has the specific value of $p_{i}=1 / i$ for $i \in \mathcal{N}$ (see, e.g. [10]).

After Bruss [3], which includes the problem with a random number of observations, the odds theorem has been extended in several directions. Bruss and Paindaveine [5] extended it to the problem of selecting the last $\ell(>1)$ successes. Hsiau and Yang [12] considered the problem with Markov-dependent trials. Recently, Ferguson [8] extended the odds theorem in several ways, where an infinite number of trials are allowed, the payoff for not selecting till the end is different from the payoff for selecting a success that is not the last, and the trials are generally dependent. Furthermore, he applied his extension to the stopping game of Sakaguchi [14].

In this paper we consider yet another extension of the result by Bruss [3]; that is, we are interested in the problem with multiple selection chances. In our first main result, we derive the optimal rule for the problem of selecting the last success with $m(\in \mathcal{N})$ selection chances and express the optimal rule as a combination of multiple odds-sums. Our extension is applied to the multi-selection versions of the problems to which the odds theorem can be applied (see, e.g. the CSP with multiple selection chances in [9] and [13]). In our second main result, we provide a formula for computing the probability of win for the problem with $m(\in \mathcal{N})$ selection chances and provide the closed-form formulae for $m=2$ and 3. Furthermore, we give the lower and upper bounds for the maximum probability of win for $m=2$ and derive its limit as $N \rightarrow \infty$ under some condition on $p_{i}, i \in \mathcal{N}$. This limit of the maximum probability of win is consistent with the known limit $\mathrm{e}^{-1}+\mathrm{e}^{-3 / 2}$ for the CSP with two selection chances (see, e.g. [1], [2], and [9]).

This paper is organized as follows. In Section 2 we consider the optimal rule for the problem of selecting the last success with $m(\in \mathcal{N})$ selection chances. Our approach is essentially based on the technique of Ano and Ando [1], in which they studied the condition for the monotone (equivalent, one-step look-ahead) selection rule to be optimal in multiple selection problems. For more details on the monotone selection problem, we refer the reader to [6] or [7]. In Section 3 we derive some formulae for the maximum probability of win. We give the bounds for the maximum probability of win for $m=2$ and derive its limit as $N \rightarrow \infty$ under some condition on $p_{i}, i \in \mathcal{N}$. Finally, we conclude the paper by making conjectures on the limits of the maximum probability of win for $m \geq 3$ and on the lower bound for $m \geq 2$. 


\section{Multiple sum-the-odds theorem}

Suppose that we are given $m(\in \mathcal{N})$ selection chances in the problem described in the preceding section. Let $V_{i}^{(m)}, i \in \mathcal{N}$, denote the conditional maximum probability of win provided that we observe $X_{i}=1$ and select this success when we have at most $m$ selection chances left. Let $W_{i}^{(m)}, i \in \mathcal{N}$, denote the conditional maximum probability of win provided that we observe $X_{i}=1$ and ignore this success when we have at most $m$ selection chances left. Furthermore, let $M_{i}^{(m)}, i \in \mathcal{N}$, denote the conditional maximum probability of win provided that we observe $X_{i}=1$ and decide whether to select when we have at most $m$ selection chances left. The optimality equation for each $m \in \mathcal{N}$ is then given by

$$
M_{i}^{(m)}=\max \left\{V_{i}^{(m)}, W_{i}^{(m)}\right\}, \quad i \in \mathcal{N} .
$$

Clearly, if $m>N-i$ (the remaining selection chances are more than the remaining observations) and we observe $X_{i}=1$, then the decision to select results in win with probability 1 , so that $M_{i}^{(m)}=V_{i}^{(m)}=1$ for $i>N-m$. In particular, we have $M_{N}^{(m)}=V_{N}^{(m)}=1$ and $W_{N}^{(m)}=0$ for any $m \in \mathcal{N}$.

We observe that $V_{i}^{(m)}$ is represented as the sum of two conditional probabilities: the first is that no success appears in $i+1, \ldots, N$ provided that $X_{i}=1$ and the second is that we finally win when starting at $i+1$ with $m-1$ selection chances provided that $X_{i}=1$. Since the latter conditional probability is equal to $W_{i}^{(m-1)}$, we have, for each $m \in \mathcal{N}$,

$$
\begin{aligned}
V_{i}^{(m)} & =\mathrm{P}\left(X_{i+1}=X_{i+2}=\cdots=X_{N}=0 \mid X_{i}=1\right)+W_{i}^{(m-1)} \\
& =\prod_{j=i+1}^{N} q_{j}+W_{i}^{(m-1)}, \quad i \in \mathcal{N}
\end{aligned}
$$

where we set $W_{i}^{(0)}:=0$ for $i \in \mathcal{N}$ and $\prod_{j=a}^{b} \cdot=1$ when $b<a$ conventionally. The second equality above follows from the independence of the $X_{i}$ s. On the other hand, $W_{i}^{(m)}$ is given as the conditional probability with which we finally win when we make the optimal decision at the first success after $i$ provided that $X_{i}=1$, so that, for each $m \in \mathcal{N}$,

$$
\begin{aligned}
W_{i}^{(m)} & =\sum_{j=i+1}^{N} \mathrm{P}\left(X_{i+1}=\cdots=X_{j-1}=0, X_{j}=1 \mid X_{i}=1\right) M_{j}^{(m)} \\
& =\sum_{j=i+1}^{N}\left(\prod_{k=i+1}^{j-1} q_{k}\right) p_{j} M_{j}^{(m)}, \quad i \in \mathcal{N} .
\end{aligned}
$$

As a preparatory step in studying the problem with multiple selection chances, we hereby provide an alternative proof of the odds theorem (Proposition 1.1) using the notion of the monotone stopping rule in [6].

An alternative proof of Proposition 1.1. We prove only the first part of Proposition 1.1. The monotone selection region for the single selection problem is given by

$$
B^{(1)}:=\left\{i \in \mathcal{N}: G_{i}^{(1)}>0\right\},
$$

where

$$
G_{i}^{(1)}:=V_{i}^{(1)}-\sum_{j=i+1}^{N}\left(\prod_{k=i+1}^{j-1} q_{k}\right) p_{j} V_{j}^{(1)}, \quad i \in \mathcal{N} .
$$


Note that $B^{(1)}$ is the region of $i \in \mathcal{N}$ such that the probability of win by selecting $X_{i}=1$ is greater than that by ignoring $X_{i}=1$ and then selecting the first success after $X_{i}$. From (2.2) we have $V_{i}^{(1)}=\prod_{j=i+1}^{N} q_{j}$, and, if there exists $j \in\{i+1, \ldots, N\}$ such that $q_{j}=0$, then (2.4) leads to $G_{i}^{(1)} \leq 0$. On the other hand, if $q_{j}>0$ for all $j=i+1, \ldots, N$ then (2.4) is written as

$$
\begin{aligned}
G_{i}^{(1)} & =\prod_{j=i+1}^{N} q_{j}-\sum_{j=i+1}^{N}\left(\prod_{k=i+1}^{j-1} q_{k}\right) p_{j}\left(\prod_{k=j+1}^{N} q_{k}\right) \\
& =\prod_{j=i+1}^{N} q_{j}\left(1-\sum_{j=i+1}^{N} r_{j}\right) .
\end{aligned}
$$

Therefore, if $G_{i}^{(1)}>0$ for some $i \in \mathcal{N}$ then $q_{j}>0$ for all $j=i+1, \ldots, N$ and (2.5) gives $\sum_{j=i+1}^{N} r_{j}<1$. Conversely, if $\sum_{j=i+1}^{N} r_{j}<1$ for some $i \in \mathcal{N}$ then $q_{j}>0$ for all $j=i+1, \ldots, N$ and (2.5) gives $G_{i}^{(1)}>0$. Namely, $G_{i}^{(1)}>0$ is equivalent to $\sum_{j=i+1}^{N} r_{j}<1$ and $B^{(1)}$ is given by

$$
B^{(1)}=\left\{i \in \mathcal{N}: \sum_{j=i+1}^{N} r_{j}<1\right\} .
$$

Since $\sum_{j=i+1}^{N} r_{j}$ is clearly nonincreasing in $i, B^{(1)}$ is 'closed' in the sense of the monotone problem in [6]; that is, $i \in B^{(1)}$ implies that $j \in B^{(1)}$ for all $j=i, i+1, \ldots, N$. Hence, the optimal rule for the single selection problem is given by (1.1) and (1.2).

We can now state the optimal rules for the multiple selection problem.

Theorem 2.1. Suppose that we have at most $m(\in \mathcal{N})$ selection chances. Then, the optimal selection rule $\tau_{*}^{(m)}$ is given by

$$
\begin{aligned}
\tau_{*}^{(m)} & =\min \left\{i \geq i_{*}^{(m)}: X_{i}=1\right\}, \\
i_{*}^{(m)} & =\min \left\{i \in \mathcal{N}: H_{i}^{(m)}>0\right\},
\end{aligned}
$$

where $\min (\varnothing)=+\infty$ and, for each $i \in \mathcal{N}$, the $H_{i}^{(m)}, m \in \mathcal{N}$, are recursively defined by

$$
\begin{aligned}
H_{i}^{(1)} & :=1-\sum_{j=i+1}^{N} r_{j}, \\
H_{i}^{(m)} & :=H_{i}^{(1)}+\sum_{j=(i+1) \vee i_{*}^{(m-1)}}^{N} r_{j} H_{j}^{(m-1)}, \quad m=2,3, \ldots, N,
\end{aligned}
$$

with $a \vee b=\max \{a, b\}$ for $a, b \in \mathbb{R}$. In (2.9), if there exists $a j \in\{i+1, \ldots, N\}$ such that $p_{j}=1$ (that is, $\left.r_{j}=+\infty\right)$, then we set $H_{i}^{(m)}:=-\infty$. Furthermore, we have

$$
1=i_{*}^{(N)} \leq i_{*}^{(N-1)} \leq \cdots \leq i_{*}^{(1)} \leq N
$$

Proof. The monotone selection region for the problem with $m(\in \mathcal{N})$ selection chances is defined by $B^{(m)}:=\left\{i \in \mathcal{N}: G_{i}^{(m)}>0\right\}$, where

$$
G_{i}^{(m)}:=V_{i}^{(m)}-\sum_{j=i+1}^{N}\left(\prod_{k=i+1}^{j-1} q_{k}\right) p_{j} V_{j}^{(m)}, \quad i \in \mathcal{N} .
$$


To derive (2.6) and (2.7), it suffices to show that $B^{(m)}$ is closed and satisfies $B^{(m)}=\{i \in$ $\left.\mathcal{N}: H_{i}^{(m)}>0\right\}$, which is also deduced by verifying that $G_{i}^{(m)}>0$ is equivalent to $H_{i}^{(m)}>0$ for each $i \in \mathcal{N}$ and that $i \mapsto H_{i}^{(m)}$ changes sign from nonpositive to positive at most once. On the other hand, to obtain (2.10), it suffices to show that $H_{i}^{(m)} \geq H_{i}^{(m-1)}$ for $i \in \mathcal{N}$ such that $H_{i}^{(m-1)}>-\infty$. We verify them by induction on $m$.

While proving Proposition 1.1, we have observed that $G_{i}^{(1)}>0$ is equivalent to $H_{i}^{(1)}>0$ for $i \in \mathcal{N}$. In particular, if $q_{j}=0$ for some $j \in\{i+1, \ldots, N\}$ then $G_{i}^{(1)} \leq 0$, while if $q_{j}>0$ for all $j=i+1, \ldots, N$ then it holds that $G_{i}^{(1)}=\left(\prod_{j=i+1}^{N} q_{j}\right) H_{i}^{(1)}($ refer to (2.5) and (2.8)). We have also observed that $i \mapsto H_{i}^{(1)}$ changes sign from nonpositive to positive at most once. The inequality $H_{i}^{(2)} \geq H_{i}^{(1)}$ for $i \in \mathcal{N}$ such that $H_{i}^{(1)}>-\infty$ is immediately obtained from (2.9); that is,

$$
H_{i}^{(2)}-H_{i}^{(1)}=\sum_{j=(i+1) \vee i_{*}^{(1)}}^{N} r_{j} H_{j}^{(1)} \geq 0,
$$

where the last inequality follows from $H_{j}^{(1)}>0$ for $j \geq i_{*}^{(1)}$.

As we apply the induction hypothesis, for $m^{\prime}=1,2, \ldots, m$ with some fixed $m \in\{1,2, \ldots$, $N-1$, we now assume the following.

(i) $G_{i}^{\left(m^{\prime}\right)}>0$ is equivalent to $H_{i}^{\left(m^{\prime}\right)}>0$ for every $i \in \mathcal{N}$. In particular, if $q_{j}=0$ for some $j \in\{i+1, \ldots, N\}$ then $G_{i}^{\left(m^{\prime}\right)} \leq 0$, and if $q_{j}>0$ for all $j=i+1, \ldots, N$ then it holds that $G_{i}^{\left(m^{\prime}\right)}=\left(\prod_{j=i+1}^{N} q_{j}\right) H_{i}^{\left(m^{\prime}\right)}$.

(ii) $i \mapsto H_{i}^{\left(m^{\prime}\right)}$ changes sign from nonpositive to positive at most once.

(iii) $H_{i}^{\left(m^{\prime}+1\right)}-H_{i}^{\left(m^{\prime}\right)} \geq 0$ for $i \in \mathcal{N}$ such that $H_{i}^{\left(m^{\prime}\right)}>-\infty$.

By the induction hypothesis, $H_{i}^{(m)}>0$ and, equivalently, $G_{i}^{(m)}>0$ for $i \geq i_{*}^{(m)}$. Thus, by (i), $q_{j}>0$ for all $j=i_{*}^{(m)}+1, \ldots, N$. Let us prove (i)-(iii) for $m^{\prime}=m+1$. We first examine (i). From (2.11), the monotone selection region in the case with $m+1$ selection chances is given by $B^{(m+1)}=\left\{i \in \mathcal{N}: G_{i}^{(m+1)}>0\right\}$, where

$$
G_{i}^{(m+1)}=V_{i}^{(m+1)}-\sum_{j=i+1}^{N}\left(\prod_{k=i+1}^{j-1} q_{k}\right) p_{j} V_{j}^{(m+1)}, \quad i \in \mathcal{N} .
$$

Since $V_{j}^{(m+1)}=V_{j}^{(1)}+W_{j}^{(m)}$ from (2.2), substituting this into (2.12), we obtain

$$
\begin{aligned}
G_{i}^{(m+1)} & =V_{i}^{(1)}+W_{i}^{(m)}-\sum_{j=i+1}^{N}\left(\prod_{k=i+1}^{j-1} q_{k}\right) p_{j}\left(V_{j}^{(1)}+W_{j}^{(m)}\right) \\
& =G_{i}^{(1)}+\sum_{j=i+1}^{N}\left(\prod_{k=i+1}^{j-1} q_{k}\right) p_{j}\left(M_{j}^{(m)}-W_{j}^{(m)}\right),
\end{aligned}
$$

where the first term on the right-hand side is obtained from (2.4) and the second term is obtained from (2.3). By the induction hypothesis we have $M_{j}^{(m)}=V_{j}^{(m)}$ for $j \geq i_{*}^{(m)}$ and $M_{j}^{(m)}=W_{j}^{(m)}$ for $j<i_{*}^{(m)}$ in $(2.1)$; that is,

$$
M_{j}^{(m)}-W_{j}^{(m)}= \begin{cases}V_{j}^{(m)}-W_{j}^{(m)} & \text { for } j \geq i_{*}^{(m)} \\ 0 & \text { for } j<i_{*}^{(m)}\end{cases}
$$


Furthermore, the induction hypothesis reads (2.3) as

$$
W_{j}^{(m)}=\sum_{\ell=j+1}^{N}\left(\prod_{k=j+1}^{\ell-1} q_{k}\right) p_{\ell} V_{\ell}^{(m)} \quad \text { for } j \geq i_{*}^{(m)}
$$

Therefore, from (2.11), we have

$$
M_{j}^{(m)}-W_{j}^{(m)}=G_{j}^{(m)} \quad \text { for } j \geq i_{*}^{(m)}
$$

Substituting this into (2.13), we have

$$
G_{i}^{(m+1)}=G_{i}^{(1)}+\sum_{j=(i+1) \vee i_{*}^{(m)}}^{N}\left(\prod_{k=i+1}^{j-1} q_{k}\right) p_{j} G_{j}^{(m)}, \quad i \in \mathcal{N} .
$$

Here, if $j \in\{i+1, \ldots, N\}$ exists such that $q_{j}=0$, then this $j$ is less than or equal to $i_{*}^{(m)}$ since $q_{j}>0$ for all $j=i_{*}^{(m)}+1, \ldots, N$. Namely, this occurs only for the case in which $i<i_{*}^{(m)}$, where the first term on the right-hand side of (2.14) is less than or equal to 0 and the second term is equal to 0 ; that is, $G_{i}^{(m+1)} \leq 0$. Conversely, suppose that $q_{j}>0$ for all $j=i+1, \ldots, N$ and some $i \in \mathcal{N}$. Then, by the induction hypothesis, applying $G_{i}^{\left(m^{\prime}\right)}=\left(\prod_{j=i+1}^{N} q_{j}\right) H_{i}^{\left(m^{\prime}\right)}$ for $m^{\prime}=1$ and $m^{\prime}=m$ to (2.14), we obtain

$$
\begin{aligned}
G_{i}^{(m+1)} & =\left(\prod_{j=i+1}^{N} q_{j}\right) H_{i}^{(1)}+\sum_{j=(i+1) \vee i_{*}^{(m)}}^{N}\left(\prod_{k=i+1}^{j-1} q_{k}\right) p_{j}\left(\prod_{\ell=j+1}^{N} q_{\ell}\right) H_{j}^{(m)} \\
& =\prod_{j=i+1}^{N} q_{j}\left(H_{i}^{(1)}+\sum_{j=(i+1) \vee i_{*}^{(m)}}^{N} r_{j} H_{j}^{(m)}\right),
\end{aligned}
$$

so that (2.9) leads to

$$
G_{i}^{(m+1)}=\left(\prod_{j=i+1}^{N} q_{j}\right) H_{i}^{(m+1)}
$$

From the observation above, if $G_{i}^{(m+1)}>0$ then $q_{j}>0$ for all $j=i+1, \ldots, N$ and (2.15) leads to $H_{i}^{(m+1)}>0$. Conversely, if $H_{i}^{(m+1)}>0$ then (2.9) states that $H_{i}^{(1)}>-\infty$; that is, $q_{j}>0$ for all $j=i+1, \ldots, N$. Thus, (2.15) also leads to $G_{i}^{(m+1)}>0$. Hence, we have (i) for $m^{\prime}=m+1$.

Next we prove (ii). By the induction hypothesis, $H_{i}^{(m+1)} \geq H_{i}^{(m)}$ for $i \in \mathcal{N}$ such that $H_{i}^{(m)}>-\infty$ and $H_{i}^{(m)}>0$ for $i \geq i_{*}^{(m)}$; that is, $H_{i}^{(m+1)}>0$ for $i \geq i_{*}^{(m)}$. For $i<i_{*}^{(m)}$, we have $\sum_{j=(i+1) \vee i_{*}^{(m)}}^{N} r_{j} H_{j}^{(m)}=\sum_{j=i_{*}^{(m)}}^{N} r_{j} H_{j}^{(m)}$, which is invariant to $i$. Thus, (2.9) states that $H_{i}^{(m+1)}\left(=H_{i}^{(1)}+\right.$ constant $)$ is nondecreasing in $i\left(<i_{*}^{(m)}\right)$. Hence, $i \mapsto H_{i}^{(m+1)}$ changes sign from nonpositive to positive at most once, and (ii) holds for $m^{\prime}=m+1$. 
Finally, to prove (iii) for $m^{\prime}=m+1$, we use (2.9) and take the difference between $H_{i}^{(m+2)}$ and $H_{i}^{(m+1)}$; that is,

$$
\begin{aligned}
H_{i}^{(m+2)}-H_{i}^{(m+1)} & =\sum_{j=(i+1) \vee i_{*}^{(m+1)}}^{N} r_{j} H_{j}^{(m+1)}-\sum_{j=(i+1) \vee i_{*}^{(m)}}^{N} r_{j} H_{j}^{(m)} \\
& \geq \sum_{j=(i+1) \vee i_{*}^{(m)}}^{N} r_{j}\left(H_{j}^{(m+1)}-H_{j}^{(m)}\right) \\
\geq & 0,
\end{aligned}
$$

where the first inequality follows from $H_{j}^{(m+1)}>0$ for $j \geq i_{*}^{(m+1)}$ and $i_{*}^{(m+1)} \leq i_{*}^{(m)}$ by the induction hypothesis. The second inequality also follows from the induction hypothesis. Hence, the induction is completed and so is the proof.

Let $h_{i}^{(m)}:=1-H_{i}^{(m)}$ for $i$ and $m \in \mathcal{N}$. From (2.9), the $h_{i}^{(m)}$ for $m \in \mathcal{N}$ are then given by

$$
\begin{aligned}
h_{i}^{(1)} & =\sum_{j=i+1}^{N} r_{j}, \\
h_{i}^{(m)} & =\sum_{j=i+1}^{i_{*}^{(m-1)}-1} r_{j}+\sum_{j=(i+1) \vee i_{*}^{(m-1)}}^{N} r_{j} h_{j}^{(m-1)}, \quad m=2,3, \ldots
\end{aligned}
$$

We can observe from the above equations that each $h_{i}^{(m)}$ is expressed as a combination of multiple odds-sums. For instance, $h_{i}^{(2)}$ and $h_{i}^{(3)}$ are calculated as

$$
\begin{aligned}
h_{i}^{(2)} & =\sum_{j=i+1}^{i_{*}^{(1)}-1} r_{j}+\sum_{j=(i+1) \vee i_{*}^{(1)}}^{N} r_{j} \sum_{k=j+1}^{N} r_{k}, \\
h_{i}^{(3)} & =\sum_{j=i+1}^{i_{*}^{(2)}-1} r_{j}+\sum_{j=(i+1) \vee i_{*}^{(2)}}^{N} r_{j}\left\{\sum_{k=j+1}^{i_{*}^{(1)}-1} r_{k}+\sum_{k=(j+1) \vee i_{*}^{(1)}}^{N} r_{k} \sum_{\ell=k+1}^{N} r_{\ell}\right\} .
\end{aligned}
$$

The optimal rule for the problem with $m(\in \mathcal{N})$ selection chances then reduces to $\tau_{*}^{(m)}=$ $\min \left\{i \in \mathcal{N}: h_{i}^{(m)}<1\right.$ and $\left.X_{i}=1\right\}$. Hence, we call Theorem 2.1 the 'multiple sum-the-odds theorem', or the 'multiple odds theorem' for short.

\section{Maximum probability of win}

In this section we first derive a formula for computing the maximum probability of win under the optimal rule with $m(\in \mathcal{N})$ selection chances and then provide closed-form formulae for $m=2$ and 3. Then, we give its lower and upper bounds and the limit as $N \rightarrow \infty$ for $m=2$.

Theorem 3.1. For the problem with at most $m(\in \mathcal{N})$ selection chances, the maximum probability of win under the optimal rule, $P^{(m)}($ win $)=P_{N}^{(m)}\left(p_{1}, \ldots, p_{N}\right)$, is given by

$$
P^{(m)}(\operatorname{win})=\prod_{j=i_{*}^{(m)}}^{N} q_{j} \sum_{j=i_{*}^{(m)}}^{N} r_{j}+\sum_{j=i_{*}^{(m)}}^{N}\left(\prod_{k=i_{*}^{(m)}}^{j} q_{k}\right) r_{j} W_{j}^{(m-1)},
$$


where if $p_{i_{*}^{(m)}}=1$ then $P^{(m)}($ win $)=\prod_{k=i_{*}^{(m)}+1}^{N} q_{k}+W_{i_{*}^{(m)}}^{(m-1)}$ (note that $p_{j}<1$ for all $\left.j=i_{*}^{(m)}+1, \ldots, N\right)$. Specifically, for $m=2$ and 3 ,

$$
\begin{aligned}
P^{(2)}(\text { win })= & \prod_{j=i_{*}^{(2)}}^{N} q_{j} \sum_{j=i_{*}^{(2)}}^{N} r_{j}\left(1+\prod_{k=j+1}^{i_{*}^{(1)}-1}\left(1+r_{k}\right) \sum_{k=(j+1) \vee i_{*}^{(1)}}^{N} r_{k}\right), \\
P^{(3)}(\text { win })= & \prod_{j=i_{*}^{(3)}}^{N} q_{j} \sum_{j=i_{*}^{(3)}}^{N} r_{j}\left[1+\prod_{k=j+1}^{i_{*}^{(2)}-1}\left(1+r_{k}\right)\right. \\
& \left.\times \sum_{k=(j+1) \vee i_{*}^{(2)}}^{N} r_{k}\left(1+\prod_{\ell=k+1}^{i_{*}^{(1)}-1}\left(1+r_{\ell}\right) \sum_{\ell=(k+1) \vee i_{*}^{(1)}}^{N} r_{\ell}\right)\right] .
\end{aligned}
$$
Proof. Note that the independence of the $X_{i}$ s leads to $P^{(m)}($ win $)=W_{i_{*}^{(m)}-1}^{(m)}$ under the
optimal selection rule. Thus, from (2.2) and (2.3), we obtain

$$
\begin{aligned}
P^{(m)}(\text { win }) & =\sum_{j=i_{*}^{(m)}}^{N}\left(\prod_{k=i_{*}^{(m)}}^{j-1} q_{k}\right) p_{j} M_{j}^{(m)} \\
& =\sum_{j=i_{*}^{(m)}}^{N}\left(\prod_{k=i_{*}^{(m)}}^{j-1} q_{k}\right) p_{j}\left(\prod_{\ell=j+1}^{N} q_{\ell}+W_{j}^{(m-1)}\right),
\end{aligned}
$$

where the second equality follows from $M_{j}^{(m)}=V_{j}^{(m)}$ for $j \geq i_{*}^{(m)}$. Hence, (3.1) is easily obtained.

The probabilities $P^{(2)}$ (win) and $P^{(3)}$ (win) are derived from straightforward calculations. Since the optimal rule requires the selection of the first success after $i_{*}^{(1)}$, we have $M_{k}^{(1)}=$ $V_{k}^{(1)}=\prod_{\ell=k+1}^{N} q_{k}$ for $k \geq i_{*}^{(1)}$. It then follows from (2.3) that

$$
W_{j}^{(1)}=\sum_{k=j+1}^{N}\left(\prod_{\ell=j+1}^{k-1} q_{\ell}\right) p_{k} M_{k}^{(1)}=\prod_{\ell=j+1}^{N} q_{\ell} \sum_{k=j+1}^{N} r_{k} \text { for } j \geq i_{*}^{(1)}-1 .
$$

On the other hand, for $j<i_{*}^{(1)}-1$, we have $W_{j}^{(1)}=W_{i_{*}^{(1)}-1}^{(1)}=\prod_{\ell=i_{*}^{(1)}}^{N} q_{\ell} \sum_{j=i_{*}^{(1)}}^{N} r_{j}$. Therefore, for each $j \in \mathcal{N}$,

$$
W_{j}^{(1)}=\prod_{\ell=(j+1) \vee i_{*}^{(1)}}^{N} q_{\ell} \sum_{k=(j+1) \vee i_{*}^{(1)}}^{N} r_{k} .
$$

Substituting this into (3.1) with $m=2$ and using $1 / q_{k}=1+r_{k}$ yields (3.2).

Using an approach similar to that used above, we obtain

$$
W_{j}^{(2)}=\prod_{\ell=(j+1) \vee i_{*}^{(2)}}^{N} q_{\ell} \sum_{k=(j+1) \vee i_{*}^{(2)}}^{N} r_{k}\left(1+\prod_{\ell=k+1}^{i_{*}^{(1)}-1}\left(1+r_{\ell}\right) \sum_{\ell=(k+1) \vee i_{*}^{(1)}}^{N} r_{\ell}\right) .
$$

Substituting this into (3.1) with $m=3$ yields (3.3). 
Next, we consider the lower and upper bounds for the maximum probability of win for $m=2$ and its limit as $N \rightarrow \infty$. In the following, to emphasize the dependence on $N$, we use the subscript ' $N$ ', and write $P_{N}^{(m)}$ (win) and $i_{*, N}^{(m)}$ occasionally. Let $R_{N}^{(m)}=\sum_{j=i_{*, N}^{(m)}}^{N} r_{j}$ and $R_{N}^{(m, 2)}=\sum_{j=i_{*, N}^{(m)}}^{N} r_{j}^{2}$ for $m \in \mathcal{N}$. Note from (1.1) and (2.10) that $0<\min \left(1, \sum_{j=1}^{N} r_{j}\right) \leq$ $R_{N}^{(1)} \leq R_{N}^{(2)} \leq \cdots \leq R_{N}^{(N)} \leq \sum_{j=1}^{N} r_{j}$. For the single selection problem, Bruss [3] deduced that

$$
R_{N}^{(1)} \mathrm{e}^{-R_{N}^{(1)}}<P_{N}^{(1)}(\text { win }) \leq R_{N}^{(1)} \mathrm{e}^{-R_{N}^{(1)}+R_{N}^{(1,2)}},
$$

and further proved that, if $R_{N}^{(1)} \rightarrow 1$ and $R_{N}^{(1,2)} \rightarrow 0$ as $N \rightarrow \infty$, then

$$
P_{N}^{(1)}(\text { win }) \rightarrow \mathrm{e}^{-1} \text { as } N \rightarrow \infty \text {. }
$$

For the double selection problem, we give the bounds and the limit as $N \rightarrow \infty$ for the maximum probability of win. We observe that our limit $\mathrm{e}^{-1}+\mathrm{e}^{-3 / 2}$ is the same as that for the CSP with two selection chances under a reasonable condition on $R_{N}^{(m)}$ and $R_{N}^{(m, 2)}$ as $N \rightarrow \infty$ (see, e.g. [1], [2], and [9]).

Theorem 3.2. For the maximum probability of win with $m=2$, we have

$$
\begin{aligned}
& P_{N}^{(2)}(\text { win }) \geq R_{N}^{(1)} \mathrm{e}^{-R_{N}^{(1)}}+\mathrm{e}^{-R_{N}^{(2)}}, \\
& P_{N}^{(2)}(\text { win })<R_{N}^{(1)} \mathrm{e}^{-R_{N}^{(1)}+R_{N}^{(1,2)}}+\left(1+r_{i_{*}^{(1)}} R_{N}^{(1)}+r_{i_{*}^{(2)}}\right) \mathrm{e}^{-R_{N}^{(2)}+R_{N}^{(2,2)},}
\end{aligned}
$$

where the inequality in (3.4) becomes strict when there is at least one $i \in \mathcal{N}$ such that $p_{i}>0$. Furthermore, if $R_{N}^{(1)} \rightarrow 1, R_{N}^{(2)} \rightarrow \frac{3}{2}, R_{N}^{(1,2)} \rightarrow 0$, and $R_{N}^{(2,2)} \rightarrow 0$ as $N \rightarrow \infty$, then

$$
P_{N}^{(2)}(\text { win }) \rightarrow \mathrm{e}^{-1}+\mathrm{e}^{-3 / 2} \text { as } N \rightarrow \infty \text {. }
$$

Proof. We first derive the lower bound of (3.4). A simple expansion of (3.2) in Theorem 3.1 yields

$$
\begin{aligned}
P^{(2)}(\text { win })= & R^{(2)} \prod_{j=i_{*}^{(2)}}^{N} q_{j}+R^{(1)} \sum_{j=i_{*}^{(2)}}^{i_{*}^{(1)}-1}\left(\prod_{k=i_{*}^{(2)}}^{j-1} q_{k}\right) p_{j}\left(\prod_{k=i_{*}^{(1)}}^{N} q_{k}\right) \\
& +\prod_{j=i_{*}^{(2)}}^{N} q_{j} \sum_{j=i_{*}^{(1)}}^{N} r_{j} \sum_{k=j+1}^{N} r_{k},
\end{aligned}
$$

where the subscript ' $N$ ' is omitted to simplify the notation. In the second term on the right-hand side (RHS) above, we note that $\sum_{j=i_{*}^{(2)}}^{i_{*}^{(1)}-1}\left(\prod_{k=i_{*}^{(2)}}^{j-1} q_{k}\right) p_{j}=1-\prod_{j=i_{*}^{(2)}}^{i_{*}^{(1)}-1} q_{j}$ since it represents the probability that at least one success appears from $i_{*}^{(2)}$ to $i_{*}^{(1)}-1$ when $i_{*}^{(1)}>i_{*}^{(2)}$ (while it is equal to 0 when $i_{*}^{(1)}=i_{*}^{(2)}$ ). Thus, we obtain

$$
\begin{aligned}
\text { second term on the RHS of (3.7) } & =R^{(1)}\left(1-\prod_{j=i_{*}^{(2)}}^{i_{*}^{(1)}-1} q_{j}\right) \prod_{k=i_{*}^{(1)}}^{N} q_{k} \\
& =R^{(1)}\left(\prod_{j=i_{*}^{(1)}}^{N} q_{j}-\prod_{j=i_{*}^{(2)}}^{N} q_{j}\right) .
\end{aligned}
$$


Consider the third term on the right-hand side of (3.7). Since $h_{i}^{(2)}=1-H_{i}^{(2)} \geq 1$ for $i<i_{*}^{(2)}$, substituting $i=i_{*}^{(2)}-1$ into (2.16), we have $\sum_{j=i_{*}^{(2)}}^{i_{*}^{(1)}-1} r_{j}+\sum_{j=i_{*}^{(1)}}^{N} r_{j} \sum_{k=j+1}^{N} r_{k} \geq 1$, which is equivalent to

$$
\sum_{j=i_{*}^{(1)}}^{N} r_{j} \sum_{k=j+1}^{N} r_{k} \geq 1+R^{(1)}-R^{(2)}
$$

Therefore, we obtain

$$
\text { third term on the RHS of }(3.7) \geq\left(1+R^{(1)}-R^{(2)}\right) \prod_{j=i_{*}^{(2)}}^{N} q_{j} .
$$

Substituting (3.8) and (3.9) into (3.7) yields

$$
P^{(2)}(\text { win }) \geq R^{(1)} \prod_{j=i_{*}^{(1)}}^{N} q_{j}+\prod_{j=i_{*}^{(2)}}^{N} q_{j}
$$

Here, noting that $1 / q_{j}=1+r_{j}$ and taking the logarithm, we have, for any $s \in \mathcal{N}$,

$$
\log \prod_{j=s}^{N} q_{j}=-\sum_{j=s}^{N} \log \left(1+r_{j}\right) \geq-\sum_{j=s}^{N} r_{j}
$$

where the inequality follows since $\log (1+x) \leq x$ for $x \geq 0$; the equality follows only when $x=0$. Hence, we obtain $\prod_{j=s}^{N} q_{j} \geq \mathrm{e}^{-R}$ with $R=\sum_{j=s}^{N} r_{j}$, where the inequality becomes strict unless $r_{s}=r_{s+1}=\cdots=r_{N}=0$. Substituting this into (3.10) with $s=i_{*}^{(1)}$ and $s=i_{*}^{(2)}$ yields (3.4).

Next we derive the upper bound of (3.5). For this, we examine the third term on the righthand side of (3.7). Since $h_{i}^{(2)}<1$ for $i \geq i_{*}^{(2)}$, substituting $i=i_{*}^{(2)}$ into (2.16), we obtain $\sum_{j=i_{*}^{(2)}+1}^{i_{*}^{(1)}-1} r_{j}+\sum_{j=\left(i_{*}^{(2)}+1\right) \vee i_{*}^{(1)}}^{N} r_{j} \sum_{k=j+1}^{N} r_{k}<1$, so that

$$
\sum_{j=i_{*}^{(1)}}^{N} r_{j} \sum_{k=j+1}^{N} r_{k}<1+\left(1+r_{i_{*}^{(1)}}\right) R^{(1)}-\left(R^{(2)}-r_{i_{*}^{(2)}}\right)
$$

Therefore, we obtain

$$
\text { third term on the RHS of }(3.7)<\left(1+\left(1+r_{i_{*}^{(1)}}\right) R^{(1)}-R^{(2)}+r_{i_{*}^{(2)}}\right) \prod_{j=i_{*}^{(2)}}^{N} q_{j} .
$$

Applying (3.8) and (3.11) to (3.7), we obtain

$$
P^{(2)}(\text { win })<R^{(1)} \prod_{j=i_{*}^{(1)}}^{N} q_{j}+\left(1+r_{i_{*}^{(1)}} R^{(1)}+r_{i_{*}^{(2)}}\right) \prod_{j=i_{*}^{(1)}}^{N} q_{j}
$$


Here, since $1 / q_{j}=1+r_{j}$, using $\log (1+x) \geq x-x^{2}$ for $x \geq 0$, we obtain, for any $s \in \mathcal{N}$,

$$
\log \prod_{j=s}^{N} q_{j} \leq-\sum_{j=s}^{N} r_{j}+\sum_{j=s}^{N} r_{j}^{2}
$$

Hence, by assigning $\sum_{j=s}^{N} r_{j}=R$ and $\sum_{j=s}^{N} r_{j}^{2}=R^{\prime}$, we obtain $\prod_{j=s}^{N} q_{j} \leq \mathrm{e}^{-R+R^{\prime}}$. Applying this in (3.12) with $s=i_{*}^{(1)}$ and $s=i_{*}^{(2)}$, we obtain (3.5).

Finally, we have $r_{i_{*, N}^{(1)}} \rightarrow 0$ and $r_{i_{*, N}^{(2)}} \rightarrow 0$ as $N \rightarrow \infty$, since $R_{N}^{(1,2)} \rightarrow 0$ and $R_{N}^{(2,2)} \rightarrow 0$ as $N \rightarrow \infty$, respectively. Therefore, (3.4) and (3.5) yield (3.6) as $N \rightarrow \infty$.

As a final remark, in the multiple selection problem, we make two conjectures on the limits and lower bounds for the maximum probability of win. First, if we conjecture that $R_{N}^{(m)}$ and $R_{N}^{(m, 2)}, m=1,2, \ldots$, have the same limits as those for the CSP with multiple selection chances, then the limit of the maximum probability of win is also consistent with that for the CSP; that is,

$$
\lim _{N \rightarrow \infty} P_{N}^{(m)}(\text { win })=\lim _{N \rightarrow \infty} \sum_{j=1}^{m} \frac{i_{*}^{(j)}}{N} \quad \text { for } m=1,2, \ldots
$$

The case in which $m=1$ was solved by Bruss [3] and the case in which $m=2$ is solved above. For instance, for the triple selection problem, our conjecture states that, if $R_{N}^{(1)} \rightarrow 1$, $R_{N}^{(2)} \rightarrow \frac{3}{2}$, and $R_{N}^{(3)} \rightarrow \frac{47}{24}$ with $R_{N}^{(m, 2)} \rightarrow 0, m=1,2,3$, as $N \rightarrow \infty$, then

$$
\lim _{N \rightarrow \infty} P_{N}^{(3)}(\text { win })=\mathrm{e}^{-1}+\mathrm{e}^{-3 / 2}+\mathrm{e}^{-47 / 24} \text {. }
$$

On performing some delicate and complicated calculations, this triple selection case could be confirmed by an approach similar to that for $P_{N}^{(2)}$ (win). However, the problem of general $m$ is more challenging.

Second, for the lower bounds for the maximum probability of win, our conjecture is stated as follows: for some reasonable condition on $p_{i}, i \in \mathcal{N}$,

$$
P_{N}^{(m)}(\text { win })>\lim _{N \rightarrow \infty} \sum_{j=1}^{m} \frac{i_{*}^{(j)}}{N} \quad \text { for } m=1,2, \ldots
$$

For this problem, the case in which $m=1$ was solved by Bruss [4]. However, the case in which $m=2$ is still open.

\section{Acknowledgement}

The authors wish to thank the anonymous referee for his/her helpful suggestions and comments.

\section{References}

[1] Ano, K. And Ando, A. (2000). A note on Bruss' stopping problem with random availability. In Game Theory, Optimal Stopping, Probability and Statistics (IMS Lecture Notes Monogr. 35), Institute for Mathematical Statistics, Beachwood, OH, pp. 71-82.

[2] Bruss, F. T. (1988). Invariant record processes and applications to best choice modelling. Stoch. Process. Appl. 30, 303-316.

[3] Bruss, F. T. (2000). Sum the odds to one and stop. Ann. Prob. 28, 1384-1391. 
[4] Bruss, F. T. (2003). A note on bounds for the odds theorem of optimal stopping. Ann. Prob. 31, 1859-1861.

[5] Bruss. F. T. and Paindaveine, D. (2000). Selecting a sequence of last successes in independent trials. J. Appl. Prob. 37, 389-399.

[6] Chow, Y. S., Robbins, H. And Siegmund, D. (1971). Great Expectations: The Theory of Optimal Stopping. Houghton Mifflin, Boston, MA.

[7] Ferguson, T. S. (2006). Optimal Stopping and Applications. Available at http://www.math.ucla.edu/ tom/ Stopping/Contents.html.

[8] Ferguson, T. S. (2008). The sum-the-odds theorem with application to a stopping game of Sakaguchi. Preprint.

[9] Gilbert, J. P. And Mosteller, F. (1966). Recognizing the maximum of a sequence. J. Amer. Statist. Assoc. 61, 35-73.

[10] Hill, T. P. And KRengel, U. (1992). A prophet inequality related to the secretary problem. In (Contemp. Math. 125), eds F. T. Bruss, T. S. Ferguson and S. M. Samuels, American Mathematical Society, Providence, RI, pp. 209-215.

[11] Hsiau, S.-R. And Yang, J.-R. (2000). A natural variation of the standard secretary problem. Statist. Sinica 10, 639-646.

[12] Hsiau, S.-R. and Yang, J.-R. (2002). Selecting the last success in Markov-dependent trials. J. Appl. Prob. 39, 271-281.

[13] Sakaguchi, M. (1978). Dowry problems and OLA policies. Rep. Statist. Appl. Res. Union Japan. Sci. Eng. 25, 24-28.

[14] SAKAGUCHI, M. (1984). Bilateral sequential games related to the no-information secretary problem. Math. Japon. 29, 961-973. 